\title{
Mecanismos de Coordenação na Formação de Redes de Cooperação: Associação dos Produtores de Vinhos Finos do Vale dos Vinhedos (APROVALE)
}

\author{
Coordination Mechanisms on Formation of Cooperation \\ Networks: Vale dos Vinhedos Wine Producers Association
}

\begin{abstract}
Claudio Zancan
Professor da Faculdade de Ecomomia, Administração e Contabilidade na Universidade Federal de Alagoas - Maceió - AL - Brasil.

E-mail: claudiozancan@gmail.com
\end{abstract}

Paulo da Cruz Freire dos Santos

Professor na Universidade Federal de Alagoas - Maceió - AL - Brasil. E-mail: paulodacruzfreire@gmail.com

Nicholas Joseph Tavares da Cruz

Professor na Universidade Federal de Alagoas - Maceió - AL - Brasil. E-mail: admnicholas@gmail.com

\section{Resumo}

Neste texto são apresentadas e analisadas as características estruturais e os mecanismos de coordenação envolvidos na formação da rede de cooperação Associação dos Produtores de Vinhos Finos do Vale dos Vinhedos. O quadro teórico apresenta abordagens econômicas $e$ organizacionais. O método compreendeu dois estudos: pesquisa documental e entrevistas. A rede foi formada por sete vinícolas similares e adotou quatro tipos de relacionamentos de alta densidade. Seus mecanismos de coordenação, gerenciamento da mobilidade do conhecimento e estabilidade foram importantes no processo de formação da rede, resultando na criação de uma estrutura organizacional inédita para os associados. Conclui-se sobre a importância da consideração de mecanismos de coordenação de recursos no processo estratégico de formação de redes via atores centrais, englobando relacionamentos interorganizacionais relacionados à transferência de conhecimentos e a criação de inovações. Estudos futuros abordando análise de outros momentos evolutivos desta rede, bem como de relacionamentos presentes em diferentes contextos, são recomendados.

Palavras-chave: Mecanismos de Coordenação. Redes de Cooperação. Inovação.

\section{Abstract}

This study aimed to identify and analyze structural features and coordination mechanisms involved on formation of Vale dos Vinhedos Wine Producers Association (APROVALE). The literature review adressed organizational and economic approaches. The research methodology used two studies: document analysis and interviews. The structural results show that the APROVALE was formed by seven like wineries through four type high density relationships. Coordination mechanisms, mobility management and of knowledge had importance for the success of the network formation resulting in the creation of a new organizational structure for its members. The conclusion is: the resources mechanisms coordination was important in strategic process of networking via central actors. This process involved inter-organizational relationships related to knowledge transfer and the creation of innovations. Further studies are recommended addressing other phases of the lifecycle of the network and external action of these relationships in different contexts.

Key words: Coordination Mechanisms. Cooperation Network. Innovation. 


\section{INTRODUÇÃO}

Com base em intensas transformações nos sistemas econômico e social, especialmente aquelas envolvendo estruturas produtivas regionais, para que organizações atuantes nestes contextos atinjam maior competitividade, verifica-se a necessidade contínua de adaptações nos relacionamentos interorganizacionais. Nesse processo, cada vez mais o desafio aos gestores parece ser a busca por diferenciação nas atividades estabelecidas, o que remete à consideração de novas formas relacionais capazes de desenvolver uma maior agregação de valor para os consumidores, organizações e mercados. (BRASS et al., 2004)

Nessa linha, estudos internacionais como os de Jarillo (1988), Ring e Van de Ven (1994), Human e Provan (1997), Oliver e Ebers (1998), Thompson (2003), entre outros, e nacionais, entre eles: Balestrin, Verschoore e Reyes Jr. (2010), Magalhães, Daut e Phonlor (2009), Hoffman, Molina-Morales e Martínez-Fernandez (2007), demonstram a importância das redes de cooperação como estratégias relacionais capazes de propiciar a geração de resultados que transcendem a simples soma dos recursos organizacionais individuais. Além disso, o compartilhamento de recursos e riscos, a sinergia resultante da interação organizacional e a estrutura de relacionamentos produzida, proporcionam uma configuração de elementos que poderá resultar em aumento de competitividade para organizações que estabelecem redes de cooperação como alternativa de desenvolvimento. (POWELL, 1998)

Contudo, para alguns estudiosos, entre eles, Knoke (1994), Hacki e Lighton (2001), o sucesso da formação das redes de cooperação de forma coordenada está relacionado com o papel desempenhado por meio de atores centrais envolvidos na gestão dos relacionamentos interorganizacionais desenvolvidos na rede. Na visão desses autores, as organizações centrais, denominadas hub firms, obtêm sucesso na orientação das trajetórias de desenvolvimento das redes com uso da autoridade e do poder hierárquico, possibilitando com isso, a consecução de objetivos comuns que visam evolução das redes de cooperação a partir da expansão coordenada de sua estrutura física.

No entanto, apesar dos aspectos práticos que envolvem o uso da autoridade e do poder hierárquico na coordenação dos recursos que possibilitam a formação das redes de cooperação, Grandori (1997) alerta sobre a importância da consideração de outros elementos que vão além da ênfase dada na expansão da estrutura física das redes como elementos de sucesso de seu processo criativo. De acordo com esses autores, o pressuposto básico da cooperação é a existência de equilibrio entre as partes, portanto, o nascimento das redes engloba, sobretudo, características mais sistêmicas, como aquelas que envolvem a capacidade de superação de limitações internas de seus componentes (tamanho das organizações, disponibilidade de recursos, tempo de existência, estratégias utilizadas, modelos de gestão) e externas, encontradas nos seus ambientes de atuação (cultura, setor de atividade, nível concorrencial, comportamento dos mercados consumidores).

Por essa razão, outra perspectiva sobre a coordenação de recursos capaz de expandir com a visão que enfatiza somente a criação de uma estrutura física como elemento de sucesso se verifica no modelo apresentado por Dhanaraj e Parkhe (2006). Esses autores propuseram um modelo de coordenação de recursos para a formação de redes, no qual, a autoridade e o poder hierárquico não são os únicos elementos capazes de propiciar sucesso durante a criação delas. Para Dhanaraj e Parkhe (2006), além do gerenciamento da estrutura da rede, mecanismos que coordenam a mobilidade do conhecimento $e$ a apropriabilidade da inovação precisam ser considerados. A gestão efetiva e coordenada da ação desses três mecanismos de coordenação, para esses autores, amplia o acesso das organizações centrais sobre a gestão de recursos $e$ riscos das organizações cooperantes. Tal fato propicia, condições favoráveis para que o processo de formação das redes obtenha êxito.

Com base neste modelo de coordenação de recursos para a criação de redes de cooperação proposto por Dhanaraj e Parkhe (2006), tem-se a definição do objetivo geral deste trabalho que é: identificar e analisar características estruturais e mecanismos de coordenação envolvidos na formação da rede de cooperação Associação dos Produtores de Vinhos Finos do Vale dos Vinhedos (APROVALE). É importante destacar que para a verificação dos fatores contidos no modelo de coordenação analisado, torna-se vital para a consecução do estudo, a escolha de uma rede de cooperação que possibilite contemplar características de uma trajetória de evolução coordenada durante seu ciclo 
de vida. Portanto, a escolha da rede de cooperação APROVALE foi efetuada por acreditar que a ela contém elementos evolutivos que possibilitem essa análise.

Vale ressaltar que a rede APROVALE surgiu no cenário nacional em 1995 como uma associação comercial formada por um pequeno grupo de produtores localizados na região de Bento Gonçalves, no estado do Rio Grande do Sul. O surgimento dessa associação aconteceu diante de dificuldades encontradas por esses produtores na geração individual de valor durante a produção e comercialização de vinhos finos e espumantes destinados ao mercado consumidor brasileiro. Com isso, esses produtores, caracterizados por pequenas empresas, a maioria de gestão familiar, com baixo investimento tecnológico, baixas capacidades produtivas e de investimentos, precisavam mudar as práticas de condução de suas organizações, tanto em termos produtivos como de gestão. A alternativa estratégica de criação de uma rede, que permitia o compartilhamento de recursos e riscos, parecia ser a solução ideal.

No final dos anos 2000, após esforços concentrados e coordenados por um grupo de atores centrais, a rede APROVALE é destaque nacional e internacional na criação de valor no setor vitivinícola brasileiro. Essas características indicam, dessa maneira, que a estrutura de cooperação estabelecida por essa rede representa um caso de sucesso no processo de formação de redes de cooperação capaz de apresentar um contexto prático propício para a verificação dos pressupostos teóricos trazidos no modelo proposto por Dhanaraj e Parkhe (2006). Uma vez que essa rede, no período compreendido entre 1995 e 2010, apresentou uma história de cooperação interorganizacional marcada por uma trajetória evolutiva gerida por atores centrais de forma coordenada, concebida e orientada a partir de demandas contidas no seu contexto de inserção, tais como aspectos relacionados com variáveis culturais, tecnológicas e econômicas. Merecedora, portanto, de esforços de pesquisa orientados em torno da compreensão do seu processo de formação.

\section{Redes de Cooperação}

Podolny e Stuart (1995) defiram redes de cooperação como um conjunto de mais de dois atores que mantêm contatos repetidos e frequentes uns com os outros, conservando autonomia e autoridade para resolver problemas decorrentes da cooperação. Todeva (2006), por sua vez, definiu redes de cooperação como o conjunto de transações repetidas e sustentadas por configurações relacionais e estruturais dotadas de fronteiras dinâmicas e elementos interconectados. $\mathrm{Na}$ sua concepção, redes de cooperação são percebidas como um grupo de organizações com objetivos comuns, formalmente relacionadas, com prazo ilimitado de existência, de escopo múltiplo de atuação, na qual cada membro possui individualidade legal, participa diretamente das decisões, dividindo simetricamente com os demais, benefícios e ganhos alcançados pelos esforços coletivos.

Nessa mesma linha, Balestrin, Verschoore e Reyes Jr. (2010) definiram redes de cooperação interorganizacionais como estruturas resultantes do relacionamento cooperado entre empresas, com ênfase no enfoque coletivo em substituição ao individual. Nesses termos, redes podem ser consideradas como entidades complexas, definidas como uma configuração particular de organização, cujo desempenho depende, de um lado, da capacidade de facilitar a comunicação entre seus componentes e de outro, da coerência entre os objetivos da rede e os objetivos de seus componentes.

Assim, pode-se concluir que redes de cooperação são arranjos organizacionais únicos, com estrutura formal própria, com mecanismos de coordenação específicos, relações de propriedade singulares e práticas de cooperação características que transcendem esforços individuais. Essas redes têm como propósitos a convergência de atributos que permitem uma adequação do ambiente competitivo em uma estrutura dinâmica, sustentada por ações uniformizadas, porém descentralizadas, capaz de possibilitar ganhos de escala, evitando que organizações envolvidas percam flexibilidade (THOMPSON, 2003). Exemplos desses propósitos mencionados podem envolver o compartilhamento de canais de distribuição de produtos, informação, influência política, mercadológica, estruturas de produção, comercialização e outras atividades do processo. (HAVNES; SENNESETH, 2001)

Ahlstrom-Soderling (2003) apresenta dois grupos de objetivos que envolvem a formação das redes de cooperação: redes estratégicas e redes de desenvolvimento. As redes estratégicas são aquelas que cooperam 
em atividades como marketing, vendas, compras, administração e produção. A maioria dessas redes é do tipo horizontal, ou seja, em princípio formadas por organizações que atuam no mesmo elo da cadeia de valor. As redes de desenvolvimento, por sua vez, são formadas com objetivos de qualificar os recursos dos que cooperam, desenvolvendo produtos, tecnologias, aumentando a qualidade, habilidades de produção e na troca de informações. Esses dois tipos de redes não são excludentes, uma rede estratégica pode desenvolver novos produtos, assim como as redes de desenvolvimento podem se tornar, gradativamente, uma rede estratégica. Na visão de Winkler (2006), nas redes de cooperação, organizações cooperadas possuem um objetivo em comum e decidem trabalhar juntas, trocando ideias, conhecimentos e tecnologias, contudo, mantendo autonomia na condução estratégica dos seus negócios.

Human e Provan (1997) destacam quatro objetivos estratégicos para as pequenas empresas organizadas em redes de cooperação: fluxos de informação e materiais, credibilidade organizacional, acesso em novos recursos e ganhos econômicos. Para Havnes e Senneseth (2001) as redes de cooperação proporcionam acesso à informação, recursos, mercados, tecnologias, vantagens em aprendizado, economias de escala e escopo, permitindo, além disso, que organizações associadas atinjam objetivos estratégicos, como compartilhar riscos e terceirizar funções organizacionais. Verschoore e Balestrin (2008) afirmam que o ganho competitivo das redes de cooperação possui relação significativa com os seguintes fatores em ordem decrescente de importância: provisão de soluções, ganhos de escala, de poder de mercado, aprendizagem, inovação, relações sociais, redução de custos e riscos.

Por outro lado, as redes de cooperação apresentam problemas, custos e riscos. Para Ebers e Jarillo (1997) alguns problemas que podem ocorrer são: paralisia da rede em função do número de cooperados, lentidão na tomada de decisão, coordenação, disputas de poder, falta de compromisso e desconfiança. Quanto aos principais custos envolvidos nas redes de cooperação, Boehs e Segatto-Mendes (2007) apresentam custos de formar ou romper a rede, além de manter e mudar a rede quando ela atinge a maturidade. No que diz respeito aos riscos para os relacionamentos cooperados constituídos, Grandori (1997) se refere à influência do contexto social, no qual, as organizações estão inseridas no comportamento e no desempenho das redes de cooperação. No entanto, a confiança nas inter-relações entre atores é um dos fatores que promove a redução de riscos envolvidos em transações estabelecidas, tornando a existência da rede economicamente possível (JARILLO, 1988). Conforme Poldony e Stuart (1999), para que haja uma efetiva colaboração nas redes de cooperação é necessário que atores compartilhem valores, identidades e ideologias.

Assim, considerando que redes de cooperação podem ter como objetivos o desenvolvimento das organizações que as constituem, bem como o da própria rede e, ainda, que tais objetivos podem se transformarem de acordo com os tipos de relacionamentos estabelecidos durante a trajetória evolutiva da rede, serão discutidas, no próximo tópico deste texto, as fases que compreendem a evolução das redes por meio de mecanismos de coordenação, enfatizando o processo de formação.

\section{Fases Evolutivas e Mecanismos de Coordenação na Formação de Redes de Cooperação}

No campo econômico e organizacional, os estudos que consideram redes de cooperação como objetos de análise propõem diferentes momentos da variável temporal em torno das trajetórias evolutivas das redes. Ou seja, tais estudos tratam desde o período anterior à formação da rede até o momento de sua possível dissolução. Isso significa a ocorrência de fases distintas às redes de cooperação, quer seja no âmbito individual das organizações, quer seja no desenvolvimento das redes constituídas. (RING; VAN DE VEN, 1994)

Ring e Van de Ven (1994) propuseram uma tipologia capaz de analisar as trajetórias de desenvolvimento das redes de cooperação, definindo o processo evolutivo dessas redes como um fenômeno cíclico $e$ não sequencial. As estratégias das organizações, a estrutura da indústria, o estágio tecnológico e o ciclo de vida dos produtos, para esses autores, fazem com que as redes de cooperação, continuamente, se modifiquem em um ciclo repetitivo de negociação, comprometimento e execução. Na negociação, as partes determinam, conjuntamente, oportunidades, riscos, objetivos, 
investimentos e ações que poderão ser realizadas de forma cooperada. O comprometimento se refere ao acerto entre as partes sobre o que fazer, como e quais mecanismos deverão ser utilizados para coordenar as atividades. Por fim, a execução se refere à colocação em prática dos acertos realizados nas etapas anteriores. (RING; VAN DE VEN, 1994)

Boehs e Segatto-Mendes (2007), nessa mesma linha, caracterizaram a trajetória de evolução das redes de cooperação por meio de uma perspectiva dinâmica e processual dos relacionamentos interorganizacionais, compreendida nas fases de formação, de consolidação e de evolução. Os resultados obtidos por esses autores consistiram na identificação de mecanismos de controle adotados ao longo das fases de interação da rede analisada, bem como abrangência, foco e fatores influenciadores na adoção de mecanismos de coordenação dessa rede.

Felzenstein e Gimmon (2009) ao analisarem o processo evolutivo das redes de cooperação em marketing, em diferentes regiões do mundo, concluíram que essas redes iniciam suas atividades com o estabelecimento de um processo de compras conjuntas, havendo pouca troca de informações entre os associados. Uma vez que as interações ainda são pouco intensas e por não haver histórico de relacionamentos prévios, o grau de confiança entre as partes é baixo, exigindo uma governança baseada em contratos. Com a consolidação do processo de cooperação, as organizações realizam atividades de comunicação e divulgação da marca de maneira a criar uma identidade da rede para o mercado. Isso faz com que as ações passem a concentrarem esforços, podendo alcançar custos mais competitivos. Ainda, para esses autores, a busca de inovações tecnológicas por meio do desenvolvimento de produtos conjuntos em redes de cooperação é um estágio no qual é necessário grande compartilhamento de informações e conhecimentos na área mercadológica.

Por sua vez, Franco (2007) especificou três diferentes e interrelacionadas fases que constituem uma rede de cooperação em torno de sua trajetória de evolução: formação, consolidação e desenvolvimento. $\mathrm{Na}$ fase de formação ocorre a descoberta, a exploração de oportunidades de colaboração, bem como negociações entre as empresas cooperantes. Ainda, nessa fase, a identificação inicial das oportunidades baseada na validação de ideias empresariais, objetivos claros, limi- tes de recursos dos atores (internos e externos) e uma atenção significativa na eleição e no tipo de parceiro, são aspectos que devem ser considerados na redução de potenciais dificuldades entre as partes envolvidas.

$\mathrm{Na}$ consolidação, as características e os objetivos da colaboração são determinados e os meios disponíveis para a consecução da rede são estabelecidos. No entanto, os parceiros podem ainda ter interesses divergentes, dependendo de fatores internos (dimensão, antiguidade, estratégia, caráter familiar, tecnologia, entre outros) e externos (cultura, setor de atividade, mercado, concorrência, entre outros) que envolvem a rede. Por fim, Franco (2007) considerou a fase de desenvolvimento, considerando que o sucesso, a estabilidade e os resultados da colaboração entre empresas são componentes importantes. Continuando, diz esse autor

[...] se o sucesso e a estabilidade forem alcançados em uma rede de cooperação, os parceiros competem mais eficiente e eficazmente e, por conseguinte, o desempenho, a satisfação $e$ o processo de aprendizagem serão melhores, proporcionando o desenvolvimento da rede. (FRANCO, 2007, p. 157)

Especificamente, contemplando a coordenação de recursos no processo de formação das redes de cooperação - foco deste artigo - percebe-se na literatura consultada que a dinâmica que une as fases de uma rede de cooperação e proporciona o seu desenvolvimento pode estar relacionada com mecanismos de coordenação hierárquicos que dão ênfase ao papel desempenhando por atores centrais (CHEN; LEE; TONG, 2006; WINKLER, 2006, entre outros). Esses atores centrais, também denominados de hub firms, são responsáveis pelo gerenciamento das atividades de coordenação dos recursos das redes, permitindo, com isso, o sucesso na sua formação. Por meio dessa visão, a formação de uma rede de cooperação pode ser coordenada com mecanismos que consideram o uso da autoridade e do poder hierárquico nos relacionamentos estabelecidos. No entanto, esse processo de coordenação hierárquico estabelece determinismo nas relações instituídas, resultando em explicações não tão abrangentes sobre o processo evolutivo das redes no contexto interorganizacional. (GRANDORI, 1997; LOPES, BALDI, 2009) 
Dentre autores que discutiram essa questão com abordagens alternativas, Dhanaraj e Parkhe (2006) apresentaram um modelo conceitual que compreende a autoridade e o poder hierárquico como não sendo os únicos mecanismos possíveis de coordenação capazes de propiciar o sucesso na criação das redes de cooperação. Tal fato pode ser conseguido com a utilização do gerenciamento da mobilidade do conhecimento, da apropriabilidade da inovação e da estabilidade da rede. Para esses autores, o gerenciamento da mobilidade do conhecimento é obtido por meio da absorção de conhecimento, criação de uma identidade percebida pela rede e capacidade de socialização interorganizacional. Já, o gerenciamento da apropriabilidade da inovação compreende uma distribuição equitativa dos valores criados no estabelecimento da rede por meio de processos de confiança, procedimentos justos $e$ participação do direito de propriedade da inovação.

Finalmente, Dhanaraj e Parkhe (2006) apontaram que o gerenciamento da estabilidade da rede pode ser conseguido por meio do aumento da reputação do ator central perante os outros atores, definição de uma visão de futuro para a rede e fortalecimento de relacionamentos existentes, tornando-os mais complexos. Os três mecanismos de coordenação indicados por Dhanaraj e Parkhe (2006), quando combinados, são responsáveis pela dinâmica que poderá permitir o sucesso na formação e consequente evolução das fases de uma rede de cooperação.

Assim, buscando ampliar a visão sobre os componentes envolvidos na coordenação das redes de cooperação no momento de sua formação, de forma sintetizada, é apresentado o Quadro 1. O quadro apresentado mostra alguns dos elementos condicionantes relacionados com o momento de formação das redes de cooperação relacionados com os estudos discutidos nesta revisão teórica. Tais elementos condicionantes, a partir da visão proposta neste artigo, são apresentados como importantes na análise da formação da rede de cooperação Associação dos Produtores de Vinhos Finos do Vale dos Vinhedos.

Após a apresentação do conceito de redes, suas fases e dinâmica evolutiva, com a finalidade de analisar o processo de formação da rede de cooperação APROVALE, por meio de medidas estruturais e mecanismos de coordenação, serão detalhados os procedimentos metodológicos deste estudo.

\begin{tabular}{|c|c|}
\hline $\begin{array}{l}\text { FATORES CONDICIONANTES } \\
\text { FORMADORES DAS REDES }\end{array}$ & Autores relacionados \\
\hline História da empresa & Larson (1992) \\
\hline Redes Pessoais e Sociais & $\begin{array}{l}\text { Butler, Phan e Hansen (1990), } \\
\text { Coleman (1990), Gambetta } \\
\text { (1988), Putnan (1996), Perrow } \\
\text { (1992) }\end{array}$ \\
\hline $\begin{array}{l}\text { Processos de negociação } \\
\text { formal }\end{array}$ & Ring e Van de Ven (1994) \\
\hline $\begin{array}{l}\text { Comportamentos } \\
\text { unilaterais }\end{array}$ & Ring e Van de Ven (1994) \\
\hline $\begin{array}{l}\text { Identificação e seleção de } \\
\text { parceiros }\end{array}$ & $\begin{array}{l}\text { Geringer (1991), Hermosilla e } \\
\text { Solá (1991), Chung, Singh e } \\
\text { Lee (2000) }\end{array}$ \\
\hline $\begin{array}{l}\text { Economia de escala e } \\
\text { poder de mercado }\end{array}$ & $\begin{array}{l}\text { Verschoore e Balestrin (2008), } \\
\text { Lorenzi e Baden-Fuller (1995), } \\
\text { Human e Provan (1997) e } \\
\text { Perrow (1992) }\end{array}$ \\
\hline Aprendizagem e inovação & $\begin{array}{l}\text { Verschoore e Balestrin (2008), } \\
\text { Powell (1998), Gulati (1998) e } \\
\text { Schibany e Polt (2001) }\end{array}$ \\
\hline Redução de custos e riscos & $\begin{array}{l}\text { Verschoore e Balestrin (2008), } \\
\text { Ebers e Jarillo (1997) e Jarillo } \\
\text { (1988) }\end{array}$ \\
\hline Razões estratégicas & \multirow{3}{*}{$\begin{array}{l}\text { Franco (2007), Ebers e Jarillo } \\
\text { (1997), Jarillo (1988), Lorenzoni } \\
\text { e Baden-Fuller (1995) e Human } \\
\text { e Provan (1997) }\end{array}$} \\
\hline Razões exploratórias & \\
\hline Razões não deliberadas & \\
\hline
\end{tabular}

Quadro 1: Componentes envolvidos na formação das redes de cooperação

Fonte: Elaborado pelos autores deste artigo

\section{MÉtodo}

Para atender ao objetivo principal aqui proposto - identificar e analisar características estruturais e mecanismos de coordenação envolvidos na formação da rede de cooperação Associação dos Produtores de Vinhos Finos do Vale dos Vinhedos (APROVALE) foram utilizados dois estudos: pesquisa documental e entrevistas, sendo classificado como um estudo descritivo-comparativo. O nível de análise foi o de redes e as unidades de análises, os relacionamentos cooperados estabelecidos entre organizações associadas à APROVALE e a entidades representativas da indústria brasileira de vinhos finos e espumantes durante o momento de formação da rede. 
$\mathrm{Na}$ pesquisa documental foram investigadas informações sobre o tema "Redes de Cooperação", identificadas por meio de pesquisa efetuada nas bases de dados do Business ABI/Inform Global e Business Source Complete, utilizando-se os termos: Interorganizational network e Cooperation; Social network e Cooperation network, Cooperation network e collaborative, e; Innovation network e Cooperation. Esses termos deveriam estar presentes em títulos, palavras-chave ou em abstracts de artigos completos, com dados empíricos, publicados em periódicos acadêmicos, no período de janeiro de 2000 a dezembro de 2010. Com a utilização dos mesmos critérios do levantamento anterior, com palavras-chave em português, realizou-se busca nos principais periódicos brasileiros de Administração (RAE, RAE-eletrônica, RAC, RAC-eletrônica, Rausp, $\mathrm{O} \& \mathrm{~S}$ e ReAD), bem como em teses dos programas de pós-graduação reconhecidos pela Capes com conceito igual ou superior a 5 . Além disso, foram examinados os sítios eletrônicos do Instituto Brasileiro do Vinho, da Associação dos Produtores de Vinhos Finos do Vale dos Vinhedos e da Empresa Brasileira de Pesquisa Agropecuária Uva e Vinho.

Para a análise dos dados obtidos na pesquisa documental, após uma análise detalhada dos documentos encontrados, esses dados foram agrupados e resumidos em três categorias: a vitivinicultura do cenário nacional, no estado do Rio Grande do Sul e na região do Vale dos Vinhedos. É válido ressaltar que por motivos de espaço, neste artigo, são apresentados apenas os resultados encontrados no terceiro tópico que envolveu a região do Vale dos Vinhedos, com especificidade na APROVALE. A importância da apresentação destas informações está na contextualização do cenário que envolveu a formação da rede analisada.

Na realização das entrevistas foram ouvidos nove gestores de vinícolas associadas à APROVALE, além de três representantes de organizações envolvidas com a criação da rede a respeito de questões sobre o seu processo de formação. As entrevistas foram realizadas entre os meses de novembro e dezembro de 2009. O Quadro 2 relaciona as organizações entrevistadas, os cargos dos entrevistados e a duração de cada entrevista.

\begin{tabular}{|c|c|c|}
\hline $\begin{array}{l}\text { ORGANIZAÇÃo } \\
\text { ENTREVISTADA }\end{array}$ & $\begin{array}{c}\text { CARGO DO } \\
\text { RESPONDENTE }\end{array}$ & $\begin{array}{l}\text { TEMPo dE } \\
\text { DuRAÇão }\end{array}$ \\
\hline Casa Valduga & Gerente de Marketing & 75 minutos \\
\hline Chandon do Brasil & Diretor Geral & 91 minutos \\
\hline $\begin{array}{l}\text { Cooperativa e } \\
\text { Vinícola Aurora }\end{array}$ & Gerente de Marketing & 63 minutos \\
\hline Vinícola Miolo & Gerente de Marketing & 84 minutos \\
\hline $\begin{array}{l}\text { Vallontano Vinhos } \\
\text { Nobres }\end{array}$ & $\begin{array}{l}\text { Proprietária - diretora } \\
\text { comercial }\end{array}$ & 100 minutos \\
\hline $\begin{array}{l}\text { Vinícola Boutique } \\
\text { Lídio Carraro }\end{array}$ & $\begin{array}{l}\text { Proprietária - diretora } \\
\text { comercial }\end{array}$ & 61 minutos \\
\hline Vinícola Cordellier & Diretor Industrial & 64 minutos \\
\hline $\begin{array}{l}\text { Vinícola Dom } \\
\text { Cândido }\end{array}$ & $\begin{array}{l}\text { Proprietário - diretor } \\
\text { administrativo }\end{array}$ & 68 minutos \\
\hline $\begin{array}{l}\text { Vinícola Marco } \\
\text { Luigi }\end{array}$ & $\begin{array}{l}\text { Proprietária - diretora } \\
\text { administrativa }\end{array}$ & 86 minutos \\
\hline IBRAVIN & Gerente de Marketing & 125 minutos \\
\hline $\begin{array}{l}\text { Gerência } \\
\text { Executiva } \\
\text { APROVALE }\end{array}$ & Diretor Executivo & 112 minutos \\
\hline $\begin{array}{l}\text { Embrapa Uva e } \\
\text { Vinho }\end{array}$ & $\begin{array}{l}\text { Pesquisador } \\
\text { responsável } \\
\text { APROVALE }\end{array}$ & 96 minutos \\
\hline
\end{tabular}

Quadro 2: Dados das entrevistas

Fonte: Elaborado pelos autores deste artigo

Para a análise dos dados obtidos nas entrevistas, foi utilizada a técnica de análise de conteúdo do tipo temática com codificação axial. Vale destacar que o tratamento dos dados por meio do processo de codificação axial consiste na redução de informações com bases em códigos previamente definidos constantes na literatura. Neste trabalho, os códigos foram oriundos do modelo de coordenação de redes proposto por Dhanaraj e Parkhe (2006). Assim, por meio dos softwares Ucinet e do Atlas TI, documentos primários foram preparados com a transcrição do texto das entrevistas. As unidades de significação adotadas englobaram variáveis constituídas nos três tipos de mecanismos de coordenação: mobilidade do conhecimento, apropriabilidade da inovação e estabilidade da rede. Por fim, como rótulos, foram programados os indicadores de cada modalidade de coordenação especificada. 


\section{Apresentação e Discussão de Resultados}

Neste tópico são apresentados e discutidos alguns dos principais resultados encontrados por meio da aplicação do método utilizado nesta pesquisa. Especificamente, são demonstradas características gerais do Vale dos Vinhedos e da rede APROVALE (pesquisa documental), bem como os mecanismos de coordenação que foram geridos na formação dessa rede (entrevistas).

\subsection{Características Gerais do Vale dos Vinhedos e da APROVALE}

O Vale dos Vinhedos é um território localizado na Serra Gaúcha, mais precisamente na região nordeste do estado do Rio Grande do Sul, sua composição geográfica se dá pela intersecção dos municípios de Bento Gonçalves, Garibaldi e Monte Belo do Sul. Trata-se de uma área caracterizada por uma bacia hidrográfica com vários pequenos rios e riachos que banham uma área montanhosa de $81.123 \mathrm{Km}^{2}$. A gênese de seu povoamento se deu basicamente por meio da colonização italiana por volta de 1875 . Os imigrantes italianos eram, em sua maioria, oriundos da região do Vêneto e Trento. (EMBRAPA, 2010)

Com base em dados obtidos na Empresa Brasileira de Pesquisa Agropecuária Uva e Vinho (EMBRAPA, 2010), atualmente, do total da área do Vale dos Vinhedos, $26 \%$ são encontrados vinhedos, $43 \%$ florestas e $31 \%$ está destinado para plantio de outras culturas. Ainda, com base nesses dados, tem-se que em relação ao uso do solo, $10 \%$ podem ser caracterizados como de uso tipicamente urbano em área rural. O Vale dos Vinhedos está localizado a pouco mais de $120 \mathrm{Km}$ da capital estadual do Rio Grande do Sul, Porto Alegre. Por representar um clima temperado, possui quatro estações bem definidas, sendo que sua paisagem muda em conformidade com as estações do ano. Neste sentido, a presença de um inverno rigoroso para os padrões brasileiros costuma ser explorada pela indústria do turismo instalada nas proximidades do vale. (APROVALE, 2010)

De acordo com informações extraídas do sítio eletrônico do Instituto Brasileiro do Vinho (IBRAVIN, 2010), nota-se que o território do Vale dos Vinhedos é caracterizado como um espaço da agricultura familiar. Isso é caracterizado pela existência de 375 famílias de viticultores cadastrados no Vale dos Vinhedos. Essas famílias costumam se organizar em forma de núcleos, ou seja, cada produtor se associa a uma vinícola, geralmente aquela que se encontra mais próxima da sua propriedade, fornecendo sua produção de uvas para a vinícola parceira industrializar. (IBRAVIN, 2010)

É neste cenário que surge a rede Associação dos Produtores de Vinhos Finos do Vale dos Vinhedos (APROVALE). No entanto, vale ressaltar que o processo de formação da rede não surgiu de forma espontânea entre os produtores localizados nesta região. Um dos fatores que propiciou o seu surgimento aconteceu nas décadas de 1960 e 1970, quando grandes empresas multinacionais de bebidas alcoólicas entraram no mercado brasileiro, elevando o grau de competitividade para as organizações que pertenciam ao setor vitivinícola. Nesse período, as vinícolas que estavam localizadas no Vale dos Vinhedos não estavam satisfeitas com os baixos desempenhos que envolviam seus negócios e o setor vitivinícola como um todo.

Era evidente que alguma coisa precisava ser efetuada para que os produtos provenientes desta região ganhassem competitividade e mercado. Portanto, uma associação cooperativa parecia ser uma alternativa estratégica plausível para reverter a condição precária que permeava os negócios desses produtores. Assim, em 1995, sete vinícolas fundaram a APROVALE: Vinícola Miolo Ltda., Luiz Valduga \& Filhos Ltda., Casa Cordelier Ind. de Bebidas Ltda., Vinhos Don Laurindo Ltda., Adega Cavalleri Ltda., Adega de Vinhos Finos Dom Cândido Ltda. e Vinho Casa Graciema Ltda. Nota-se na página principal do sítio eletrônico da associação APROVALE que o principal objetivo de sua criação consistiu em formar a primeira Indicação de Procedência (IP) no Brasil para vinhos finos e espumantes, bem como transformar a região do Vale dos Vinhedos em uma rota do Enoturismo nacional. (APROVALE, 2010)

Após um processo que perdurou por cerca de oito anos desde a criação da associação, em 22 de novembro de 2002, o Vale dos Vinhedos tornou-se a primeira região do Brasil a obter uma Indicação de Procedência (IP) de vinhos finos e espumantes reconhecida pela União Europeia (APROVALE, 2010). Entre os atores institucionais importantes durante o processo de reco- 
nhecimento geográfico que foram responsáveis pela elaboração e detalhamento de estudos técnicos estão: Embrapa Uva e Vinho (Empresa Brasileira de Pesquisa Agropecuária Uva e Vinho), Universidade Federal do Rio Grande do Sul (UFRGS) e Universidade de Caxias do Sul (UCS).

O titular da IP Vale dos Vinhedos é a APROVALE que, em 2010, contava com 31 vinícolas associadas e 39 associados não produtores (queijarias, hotéis, restaurantes, pousadas, entre outros). Com base na produção de 2010 (APROVALE, 2010), as vinícolas do Vale dos Vinhedos produziram um volume de 10,1 milhões de garrafas de vinhos finos e espumantes, sendo que esse volume de produção significa em torno de $20 \%$ da produção de vinhos finos e $35 \%$ da produção de espumantes, do total da produção desses produtos que são oriundos no estado do Rio Grande do Sul. (APROVALE, 2010)

Dessa maneira, é com base na importância econômica e social que essa rede representa para o setor produtivo brasileiro que os mecanismos de coordenação geridos durante sua formação ganharam relevância, podendo estes resultados inspirar a formação de outras redes produtivas no Brasil. Portanto, no próximo tópico, os resultados da análise desses mecanismos são apresentados.

\subsection{Mecanismos de Coordenação na Formação da APROVALE}

Os resultados que contêm os mecanismos de coordenação na fase de formação da APROVALE são demonstrados a seguir, especificando as medidas estruturais da rede com base nos indicadores: tamanho, diversidade dos relacionamentos, densidade, força das relações (similaridades) e centralidade dos atores. Além disso, são demonstrados os resultados que demonstram a gestão exercida pelos atores centrais sobre os mecanismos de coordenação representados por meio dos indicadores: mobilidade do conhecimento, apropriabilidade da inovação e estabilidade da rede.

\subsubsection{Medidas Estruturais da APROVALE}

Com base nas análises efetuadas nos indicadores tamanho da rede e a diversidade dos relacionamentos, tem-se que a rede APROVALE foi formada em 1995 por sete vinícolas localizadas na região do Vale dos Vinhedos, localizadas no município de Bento Gonçalves (RS), juntamente com o apoio técnico da Empresa Brasileira de Pesquisa Agropecuária Uva \& Vinho (Embrapa U\&V). Os relacionamentos predominantes estabelecidos por estas organizações durante o processo de formação da rede foram: comercial, técnico, amizade, pesquisa e desenvolvimento. O Quadro 3 resume os resultados encontrados.

\begin{tabular}{|c|c|}
\hline $\begin{array}{l}\text { Tamanho da } \\
\text { Rede }\end{array}$ & $\begin{array}{l}\text { Oito Organizações: Embrapa Uva e Vinho, } \\
\text { Vinícola Miolo, Casa Valduga, Casa } \\
\text { Graciema, Vinícola Cavalleri, Vinícola } \\
\text { Dom Cândido, Vinícola Don Laurindo e } \\
\text { Vinícola Cordelier. }\end{array}$ \\
\hline $\begin{array}{l}\text { Diversidade dos } \\
\text { Relacionamentos }\end{array}$ & $\begin{array}{l}\text { Embrapa Uva e Vinho (relacionamentos } \\
\text { técnicos e Pesquisa \& Desenvolvimento), } \\
\text { Vinícola Miolo e Casa Valduga } \\
\text { (relacionamentos comerciais, técnicos e } \\
\text { Pesquisa \& Desenvolvimento), Vinícola } \\
\text { Dom Cândido, Vinícola Cavalleri, Casa } \\
\text { Graciema, Vinícola Don Laurindo e } \\
\text { Vinícola Cordelier (relacionamentos } \\
\text { comerciais, técnicos e amizade). }\end{array}$ \\
\hline
\end{tabular}

Quadro 3: Tamanho da rede e diversidade dos relacionamentos

Fonte: Elaborado pelos autores deste artigo

Um dos fatores que merece ser destacado durante o processo de formação da rede APROVALE versa sobre os relacionamentos que começaram a ser pensados de forma cooperada entre as vinícolas. Esta questão, apesar de não ser uma novidade em uma estrutura de cooperação, era inédita para as vinícolas formadoras da rede. Nesse início, o cenário que contemplava as empresas formadoras da rede era predominado por uma visão individualista dos negócios. Portanto, a lógica da cooperação entre as organizações formadoras da APROVALE, em 1995, pode ser considerada como o fator fundamental que tornou possível o início de todo o processo. Com base nos resultados sobre o tamanho e diversidade dos relacionamentos, é possível a visualização gráfica dos relacionamentos mantidos entre os atores neste início. 


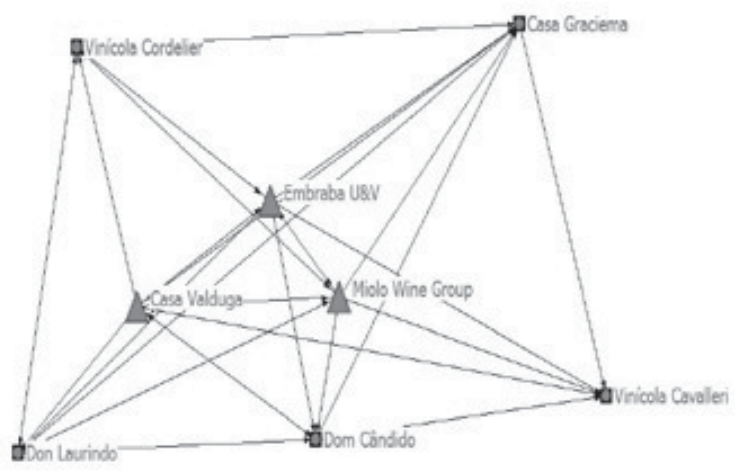

Figura 1: Representação gráfica da rede Fonte: Elaborado pelos autores deste artigo

Na Figura 1, percebe-se que apesar dos objetivos dos associados formadores da APROVALE surgirem com base em interesses comuns, na estrutura da rede criada coexistiam dois grupos de organizações: o primeiro grupo, caracterizado por um triângulo, é formado por Vinícola Miolo (Miolo Wine Group), Casa Valduga e Embrapa Uva e Vinho (Embrapa U\&V), que foram percebidas como as organizações mais envolvidas com as atividades relacionadas com a formação da rede. $\mathrm{O}$ segundo grupo, caracterizado por um quadrado com um círculo central, foi formado por: Vinícola Cavalleri, Vinícola Dom Cândido (Dom Cândido), Vinícola Don Laurindo (Don Laurindo), Vinícola Cordelier e Casa Graciema, que se comportaram como seguidoras do grupo principal.

Esses resultados demonstram que a rede APROVALE foi formada por dois grupos de organizações que assumiram importâncias distintas nas atividades de coordenação da rede. Um dos motivos que talvez possa ter levado a cooperação entre esses dois grupos foram as redes pessoais e sociais envolvidas, sendo que os gestores das vinícolas Dom Cândido e Don Laurindo possuíam parentesco em primeiro grau com o proprietário e o principal gestor da Casa Valduga. Por sua vez, o proprietário da Casa Valduga também possuía parentescos com o proprietário e principal gestor da Vinícola Miolo.

Adicionados a esses aspectos, a rede foi analisada com base nos indicadores densidade dos relacionamentos e força das ligações. Conforme contempla Scott (2000), as medidas estruturais da rede são de extrema importância na avaliação e percepção de quanto os relacionamentos são explorados. O Quadro 4 apresenta os resultados com base nesses indicadores.

\begin{tabular}{|c|c|c|}
\hline ORGANIZAÇÕES & $\begin{array}{l}\text { DensidAde } \\
\text { DA RedE }\end{array}$ & $\begin{array}{l}\text { FORÇA DAS RELAÇõES } \\
\text { (SIMILARIDADES) }\end{array}$ \\
\hline $\begin{array}{l}\text { Embrapa Uva e } \\
\text { Vinho }\end{array}$ & \multirow{8}{*}{$\begin{array}{l}0,750 \\
\text { ou } \\
75 \%\end{array}$} & \multirow{8}{*}{$\begin{array}{l}\text { Com exceção da } \\
\text { Embrapa Uva e Vinho, } \\
\text { as vinícolas formadoras } \\
\text { da rede compartilhavam } \\
\text { similaridades em tamanho } \\
\text { (pequenas empresas), } \\
\text { vínculos parentesco } \\
\text { (primos, tios, cunhados), } \\
\text { tipo de produto (vinhos } \\
\text { finos e de mesa) e técnica } \\
\text { de plantio (sistema de } \\
\text { condução latada). }\end{array}$} \\
\hline Vinícola Miolo & & \\
\hline Casa Valduga & & \\
\hline Casa Graciema & & \\
\hline $\begin{array}{l}\text { Vinícola Dom } \\
\text { Cândido }\end{array}$ & & \\
\hline $\begin{array}{l}\text { Vinícola Don } \\
\text { Laurindo }\end{array}$ & & \\
\hline Vinícola Cordelier & & \\
\hline Vinícola Cavalleri & & \\
\hline
\end{tabular}

Quadro 4: Densidade e força das relações

Fonte: Elaborado pelos autores deste artigo

Os relacionamentos estabelecidos na fase de formação da APROVALE diferiram sobre quatro conteúdos (técnico, comercial, amizade e pesquisa e desenvolvimento), resultando em uma densidade de 0,75 , ou seja, do total de 224 relacionamentos mensurados possíveis, 168 eram estabelecidos (o número de relacionamentos total é calculado levando em consideração oito organizações formadoras da rede, multiplicado pelos quatro tipos de relacionamentos identificados com cada uma). Esse resultado sobre a densidade demonstra, com base em Scott (2000), tratar-se de uma rede bastante densa. Portanto, nesta fase inicial da APROVALE, apesar de os relacionamentos possuírem limitações em termos de quantidades de conteúdos relacionais compartilhados (somente quatro), eles permitiram proximidade entre as organizações associadas à rede.

Nos resultados que contemplam a identificação da força das relações (similaridades) nas organizações envolvidas na formação da rede, percebe-se que algumas similaridades são mais evidentes, tais como: tamanho da empresa (pequenas empresas), vínculos de parentesco (primos, tios, cunhados), tipo de produtos (vinhos finos e de mesa) e técnica de plantio utilizada (sistema de condução latada). Dessa maneira, pode-se afirmar que além de densas, as relações podiam ser consideradas fortes, caracterizando com isso a existência de uma grande possibilidade dos atores formadores da APROVALE ser influenciados por padrões grupais, 
permitindo a aproximação dos interesses contidos nos dois grupos distintos de organizações identificadas durante a formação da rede.

No que tange à centralidade dos atores na rede, os resultados demonstram que a centralidade de grau de entrada e saída indicaram as organizações Vinícola Miolo e Casa Valduga como as centrais. Na centralidade de proximidade, os resultados demonstram que a Embrapa Uva e Vinho e Casa Valduga assumiram posições centrais durante a formação da APROVALE com base nesse tipo de centralidade. Isso significa que essas vinícolas centrais eram as mais próximas de todas as demais que constituíram a rede nesse início. Por sua vez, a centralidade de intermediação foi verificada em Embrapa Uva e Vinho, Casa Valduga e Vinícola Miolo, denotando com isso, centralidade dessas organizações na intermediação dos relacionamentos durante a formação da rede junto aos demais fundadores da APROVALE. Esses resultados são demonstrados no Quadro 5.

\begin{tabular}{|c|c|c|c|c|}
\hline \multirow{2}{*}{$\begin{array}{l}\text { Organiza- } \\
\text { çÕES }\end{array}$} & \multicolumn{4}{|c|}{ Centralidade } \\
\hline & ENTRADA & SAÍDA & $\begin{array}{c}\text { ProximI- } \\
\text { DAdE }\end{array}$ & $\begin{array}{c}\text { INTERME- } \\
\text { DIAÇÃo }\end{array}$ \\
\hline $\begin{array}{l}\text { Embrapa Uva } \\
\text { e Vinho }\end{array}$ & 14 & 14 & \multirow{8}{*}{$\begin{array}{l}\text { Embrapa } \\
\text { Uva e } \\
\text { Vinho } \\
\text { e Casa } \\
\text { Valduga }\end{array}$} & \multirow{8}{*}{$\begin{array}{l}\text { Embrapa } \\
\text { Uva e } \\
\text { Vinho, } \\
\text { Casa } \\
\text { Valduga } \\
\text { e Vinícola } \\
\text { Miolo }\end{array}$} \\
\hline $\begin{array}{l}\text { Vinícola } \\
\text { Miolo }\end{array}$ & 28 & 28 & & \\
\hline Casa Valduga & 28 & 28 & & \\
\hline $\begin{array}{l}\text { Casa } \\
\text { Graciema }\end{array}$ & 20 & 20 & & \\
\hline $\begin{array}{l}\text { Vinícola Dom } \\
\text { Cândido }\end{array}$ & 20 & 20 & & \\
\hline $\begin{array}{l}\text { Vinícola Don } \\
\text { Laurindo }\end{array}$ & 20 & 20 & & \\
\hline $\begin{array}{l}\text { Vinícola } \\
\text { Cordelier }\end{array}$ & 19 & 19 & & \\
\hline $\begin{array}{l}\text { Vinícola } \\
\text { Cavalleri }\end{array}$ & 19 & 19 & & \\
\hline
\end{tabular}

Quadro 5: Centralidade da rede

Fonte: Elaborado pelos autores deste artigo

Ainda sobre a centralidade da rede, vale ressaltar que a Embrapa Uva e Vinho foi apontada como a organização com maior importância pelas demais organizações, apesar de esta organização estabelecer somente dois tipos de relacionamentos (técnicos e pesquisa e desenvolvimento). $\mathrm{O}$ que denota, para esse ator, não ser uma organização central em termos de entrada e saídas de relacionamentos. No entanto, os relacionamentos mantidos pela Embrapa Uva e Vinho foram considerados pelos demais atores da rede como cruciais no início do processo de formação, pois, todos demandavam uma necessidade por melhorias técnicas e acesso a novas tecnologias para o processo o processo produtivo mantido em suas organizações. A Embrapa Uva e Vinho, devido as suas características organizacionais, preenchiam tais lacunas, por isso, era considerada central.

\subsubsection{Mecanismos de Coordenação na APROVALE}

A partir dos resultados sobre as medidas estruturais da rede que permitiram evidenciar os atores centrais da APROVALE (Embrapa Uva e Vinho, Vinícola Miolo e Casa Valduga) durante o processo de formação, são apresentados os resultados sobre o mecanismo de coordenação gerenciamento da mobilidade do conhecimento a partir da gestão dos atores centrais identificados (Quadro 6).

Com base nos resultados identificados no gerenciamento da mobilidade do conhecimento, definido por Dhanaraj e Parkhe (2006) como a capacidade de transferência de conhecimentos tácitos, é demonstrado que na Embrapa Uva e Vinho, dado o nível de qualificação dos técnicos que pertenciam ao seu corpo funcional, esse ator possuiu expressivo envolvimento nas atividades externas da rede, principalmente, na incorporação de novos conhecimentos relacionados com a formação da estrutura física da rede. Por sua vez, as vinícolas que se configuram como atores centrais, apesar de muita força de vontade e envolvimento nas atividades internas à rede, ficaram um pouco desorientadas durante o processo de transferência de conhecimentos, principalmente, a respeito de informações específicas envolvidas sobre padrões legais e técnicos, oriundas de fontes externas.

Ainda sobre o gerenciamento deste mecanismo de coordenação, percebe-se que a identidade dos atores centrais era percebida de maneira diferenciada: Embrapa Uva e Vinho constituía a percepção de uma organização com identidade forte, dado ao papel institucional representado por ela no contexto nacional; Vinícola Miolo representava uma visão empreendedora 
INDICADORES DO GERENCIAMENTO DA MOBILIDADE do Conhecimento

Nível de treinamento dos associados

(Code 1 = 16 incidências)

Nível de inserção dos associados

(Code 2 = 21 incidências)

Nível de treinamento de entidades

(Code 3 = 18 incidências)

Nível de inserção de entidades

(Code 4 = 11 incidências)

Identidade do tipo institucional

(Code $5=18$ incidências)

Identidade do tipo vínculo parentesco

(Code 6 = 21 incidências)

Identidade do tipo empreendedora

(Code 7 = 14 incidências)

Presença de universidades

(Code $9=14$ incidências)

Presença de institutos de pesquisa

(Code $10=21$ incidências)

Presença de reuniões/encontros

(Code $11=15$ incidências)

Constatação de palestras

(Code $13=18$ incidências)

Principais ASPECTOS OBSERVAdos POR ATOR CENTRAL

Embrapa Uva e Vinho

1) Alto nível de treinamento $e$ inserção do corpo funcional nas atividades externas à rede via corpo técnico da instituição.

2) Identidade Institucional forte.

3) Envolvimento com universidades e institutos de pesquisa, além da promoção de reuniões e palestras entre associados compartilhando informações técnicas.

\section{Vinícola Miolo e Casa Valduga}

1) Ambas as vinícolas detinham baixo nível de treinamento do corpo funcional em atividades internas e externas nos assuntos relacionados a gestão da rede.

2) Identidade da Casa Valduga caracterizada pelo vínculo parentesco.

3) Identidade da Vinícola Miolo caracterizada pela visão empreendedora do seu gestor.

4) Ambas as vinícolas atuavam na articulação de reuniões entre os demais associados da rede.

Quadro 6: Gerenciamento da mobilidade do conhecimento

Fonte: Elaborado pelos autores deste artigo

de sucesso junto aos demais atores, portanto, um modelo a ser seguido pelos demais, e; Casa Valduga detinha uma identidade baseada no desenvolvimento dos laços familiares mantidos com os demais atores formadores da rede.

Percebe-se com base nesses resultados que as diferenças nas características do gerenciamento da mobilidade do conhecimento entre os atores foram importantes para o sucesso da formação da rede. Uma vez que o conhecimento externo de alto nível da Embrapa Uva e Vinho possibilitou a rede na obtenção de informações técnicas junto às universidades e institutos de pesquisas. Por sua vez, tanto a Vinícola Miolo quanto a Casa Valduga exerciam uma função de socialização do conhecimento técnico obtido junto a Embrapa Uva e Vinho em um padrão de transmissão conectado com os hábitos culturais dos demais atores. Nesse sentido, esses dois atores centrais foram cruciais na promoção e articulação de reuniões com os demais associados, visando o compartilhamento dos padrões que deveriam ser adotados na rede de forma conjunta.

Abordados os resultados que envolveram o gerenciamento da mobilidade do conhecimento, tem-se neste momento a apresentação dos resultados a respeito da coordenação da apropriabilidade da inovação na fase de formação da rede. O Quadro 7 apresenta os resultados encontrados.

\begin{tabular}{|c|c|c|}
\hline \multirow{2}{*}{$\begin{array}{l}\text { INDICADORES DO } \\
\text { GERENCIAMENTO DA } \\
\text { APROPRIABILIDADE } \\
\text { DA INOVAÇÃO }\end{array}$} & \multicolumn{2}{|c|}{$\begin{array}{l}\text { PRINCIPAIS ASPECTOS OBSERVADOS POR } \\
\text { ATOR CENTRAL }\end{array}$} \\
\hline & $\begin{array}{l}\text { EMBRAPA UVA E } \\
\text { VINHO }\end{array}$ & $\begin{array}{l}\text { Vinícola Miolo } \\
\text { e Casa Valduga }\end{array}$ \\
\hline $\begin{array}{l}\text { Bases de } \\
\text { construção da } \\
\text { confiança } \\
\text { (Code } 15=24 \\
\text { incidências) } \\
\text { Comportamentos } \\
\text { unilaterais } \\
\text { (Code } 20=21 \\
\text { incidências) } \\
\text { Participação nos } \\
\text { ganhos da rede } \\
\text { (Code } 21=15 \\
\text { incidências) }\end{array}$ & $\begin{array}{l}\text { 1) Confiança } \\
\text { construída com } \\
\text { base nos processos } \\
\text { técnicos (plantio } \\
\text { e produção) } \\
\text { sugeridos e } \\
\text { implementados. } \\
\text { 2) Ausência de } \\
\text { comportamento } \\
\text { unilateral. } \\
\text { 3) Possibilidade de } \\
\text { ganhos mútuos a } \\
\text { partir das inovações } \\
\text { geradas na rede. }\end{array}$ & $\begin{array}{l}\text { 1) Confiança } \\
\text { construída com } \\
\text { base na imagem } \\
\text { do gestor } \\
\text { principal da } \\
\text { vinícola. } \\
\text { 2) Ausência de } \\
\text { comportamentos } \\
\text { unilaterais. } \\
\text { 3) Possibilidade } \\
\text { ganhos } \\
\text { individuais } \\
\text { a partir das } \\
\text { inovações } \\
\text { geradas na rede. }\end{array}$ \\
\hline
\end{tabular}

Quadro 7: Gerenciamento da apropriabilidade da inovação Fonte: Elaborado pelos autores deste artigo

Os resultados encontrados para a análise do gerenciamento da apropriabilidade da inovação na fase de formação da APROVALE podem ser interpretados com base nas influências das características institu- 
cionais presentes na Embrapa Uva e Vinho. Portanto, como esse ator central estava preocupado com o desenvolvimento econômico e social do setor vitivinícola nacional, de forma geral, as questões que envolviam a apropriabilidade da inovação ganhavam um direcionamento mais coletivista, vislumbrando interesses compartilhados entre os atores formadores da rede.

Essa condição diferiu, portanto, das visões encontradas nos demais atores centrais, que eram empresas privadas e cujo lucro financeiro com as inovações geradas na rede era crítico para o financiamento das atividades desenvolvidas ou em novas atividades planejadas. Assim, apesar de ser constatada ausência de comportamentos unilaterais entre os atores formadores da APROVALE, a possibilidade de obtenção de ganhos individuais, de maneiras diferenciadas, era uma questão que envolvia a realidade das organizações formadoras da rede desde sua criação. O comportamento de competição entre organizações concorrentes em redes é verificado, a exemplo da APROVALE, em outros estudos, como Armstrong (1997), Bengtsson e Kock (1999) e Lado, Boyd e Hanlon (1997).

Nessa direção, é importante ressaltar que na fase de formação desta rede, a maior inovação gerada foi a criação da estrutura organizacional APROVALE, ou seja, uma inovação organizacional que gerou resultados positivos para todos os atores envolvidos, sejam centrais ou não. Porém, no que se refere às inovações geradas no gerenciamento individual das organizações, dado ao comportamento de competição comercial existente entre as organizações formadoras, as inovações, na maioria das vezes, ficaram restritas aos limites da organização. Portanto, não eram compartilhadas por todos os associados da rede. Esse aspecto demonstra que a APROVALE foi constituída para que as organizações da região do Vale dos Vinhedos se associassem e ganhassem competitividade conjunta, mas não uniforme. O que não impedia que ações individuais fossem, algumas vezes, compartilhadas entre os demais atores, seja por meio de ações de benchmarking ou até mesmo em sistemas de parcerias restritas entre alguns dos associados.

Abordados os resultados que envolveram o gerenciamento da apropriabilidade da inovação, tem-se a apresentação dos aspectos referentes ao gerenciamento da estabilidade da rede na fase de formação da APROVALE. O Quadro 8 apresenta os resultados encontrados.

\begin{tabular}{|c|c|c|}
\hline \multirow{2}{*}{$\begin{array}{c}\text { INDICADORES DO } \\
\text { GERENCIAMENTO DA } \\
\text { ESTABILIDADE DA } \\
\text { REDE }\end{array}$} & \multicolumn{2}{|c|}{$\begin{array}{c}\text { PRINCIPAIS ASPECTOS OBSERVADOS POR } \\
\text { ATOR CENTRAL }\end{array}$} \\
\hline & $\begin{array}{l}\text { EMBRAPA UVA E } \\
\text { VINHO }\end{array}$ & $\begin{array}{l}\text { Vinícola Miolo } \\
\text { e Casa Valduga }\end{array}$ \\
\hline $\begin{array}{l}\text { Ator central com } \\
\text { abrangência local } \\
\text { (Code } 23=21 \\
\text { incidências) } \\
\text { Ator central com } \\
\text { abrangência regional } \\
\text { (Code } 24=18 \\
\text { incidências) } \\
\text { Ator central com } \\
\text { abrangência nacional } \\
\text { (Code } 25=6 \\
\text { incidências) } \\
\text { Definição de } \\
\text { objetivos estratégicos } \\
\text { (Code } 27=25 \\
\text { incidências) } \\
\text { Relacionamentos de } \\
\text { amizade } \\
\text { (Code } 29=15 \\
\text { incidências) } \\
\text { Relacionamentos } \\
\text { técnicos } \\
\text { (Code } 30=28 \\
\text { incidências) } \\
\text { Relacionamentos } \\
\text { comerciais } \\
\text { (Code } 31=13 \\
\text { incidências) } \\
\text { Relacionamentos } \\
\text { de Pesquisa } \\
\text { Desenvolvimento } \\
\text { (Code } 35=25 \\
\text { incidências) }\end{array}$ & $\begin{array}{l}\text { 1) Aumento da } \\
\text { reputação junto } \\
\text { ao contexto local/ } \\
\text { regional como } \\
\text { a instituição } \\
\text { relacionada aos } \\
\text { procedimentos } \\
\text { técnicos de } \\
\text { plantio e } \\
\text { produção de } \\
\text { vinhos finos e } \\
\text { espumantes. } \\
\text { 2) Estratégias } \\
\text { direcionadas } \\
\text { no aumento } \\
\text { da qualidade, } \\
\text { na busca de } \\
\text { Indicação de } \\
\text { Procedência e na } \\
\text { Denominação de } \\
\text { Origem (DOC). } \\
\text { 3) Estabelecimento } \\
\text { de } \\
\text { relacionamentos } \\
\text { técnicos (plantio, } \\
\text { produção e } \\
\text { pesquisa e } \\
\text { desenvolvimento } \\
\text { compartilhados). }\end{array}$ & $\begin{array}{l}\text { 1) Aumento de } \\
\text { reputação junto } \\
\text { ao mercado } \\
\text { consumidor } \\
\text { local/regional } \\
\text { no que se refere } \\
\text { aos aspectos } \\
\text { comerciais } \\
\text { (direcionamento } \\
\text { dos negócios) } \\
\text { e de qualidade } \\
\text { de produtos } \\
\text { oferecidos. } \\
\text { 2) Estratégias } \\
\text { direcionadas } \\
\text { no aumento } \\
\text { da qualidade, } \\
\text { na busca de } \\
\text { Indicação de } \\
\text { Procedência, na } \\
\text { Denominação } \\
\text { de Origem e } \\
\text { atividades de } \\
\text { Enoturismo. } \\
\text { 3) São } \\
\text { consolidados } \\
\text { relacionamentos } \\
\text { comerciais, } \\
\text { técnicos (plantio } \\
\text { e produção, } \\
\text { gestão e amizade. }\end{array}$ \\
\hline
\end{tabular}

Quadro 8: Gerenciamento da estabilidade da rede Fonte: Elaborado pelos autores deste artigo

Conforme pode ser percebido nos resultados apresentados para essa modalidade de gerenciamento contido no quadro acima, fica evidente a principal característica que identifica a estrutura organizacional de uma rede de cooperação: a possibilidade de estar associado e manter autonomia de interesses estratégicos inerentes ao seu negócio. Este fato explica os diferentes tipos de reputações atingidos pelos atores centrais: a) Embrapa Uva e Vinho, que aumentou sua reputação como instituição relacionada com o processo de plantio de uvas viníferas no contexto local/regional de inserção (Serra Gaúcha); b) Casa Valduga, que aumentou sua reputação junto ao mercado consumidor local/regional como organização que priorizava a qualidade e a personalização de vinhos e espumantes elaborados ; e c) Vinícola Miolo, que além do aumento 
da reputação comercial com base na qualidade dos produtos oferecidos, após a sua entrada na rede, passou ser conhecida como a "vinícola que deu certo", a partir da utilização total dos padrões de qualidade e das prerrogativas implementadas pela Embrapa Uva e Vinho na formação da APROVALE. Portanto, era um modelo de sucesso que poderia ser seguido por empresários do setor, associados ou não.

Dado as diferenças nas conduções dos negócios entre os associados à rede APROVALE, a visão de futuro definida para as organizações ganhou trajetórias que se assemelharam em alguns aspectos, em outros não. Especificamente, a Embrapa Uva e Vinho definiu a visão de futuro da APROVALE na consecução de três estágios: o primeiro estágio contou com o aumento gradual de qualidade dos produtos e serviços oferecidos na rede; o segundo estágio versou sobre a busca da Indicação de Procedência, e; o terceiro estágio foi caracterizado como a busca da Denominação de Origem Controlada Vale dos Vinhedos.

Cabe ressaltar que a visão de consecução desses estágios, que direcionaram investimentos coletivos $e$ individuais nos negócios da rede, foram compartilhados, no início, por todos os atores, sejam centrais ou não. Por sua vez, a Casa Valduga adicionou como visão de futuro à rede o estabelecimento de atividades relacionadas ao turismo, criando com isso, maiores possibilidades de incrementos nas receitas das vinícolas. Já a Vinícola Miolo visualizou o mercado internacional, percebendo com este enfoque, aumento de market-share e, consequentemente, maior possibilidade de lucratividade em seu negócio.

Com essas visões diferenciadas de futuro para a rede, os relacionamentos ganharam maiores níveis de complexidades, com isso, iniciando um processo de agregação de outras organizações do setor vitivinícola junto às possibilidades de interesses que eram contemplados. Porém, percebe-se que essa evolução foi gradual e a inserção de novos relacionamentos não implicaram na consolidação daqueles iniciais objetivados pela Embrapa Uva e Vinho junto às atividades desenvolvidas na rede. Assim, fica claro a presença de níveis de desenvolvimento diferenciados entre os atores formadores da rede, quer sejam essas diferenças referentes aos processos técnicos criados, ou ainda, relacionadas com a gestão de novos valores econô- micos que surgiram após a formalização da estrutura organizacional da APROVALE.

Em síntese, no que se refere ao gerenciamento dos mecanismos de coordenação na fase de formação da APROVALE, nota-se que os atores centrais estabeleceram o gerenciamento da mobilidade do conhecimento dando ênfase na criação de uma identidade forte percebida na rede pelas demais organizações associadas. Também, a existência de técnicos altamente capacitados na Embrapa Uva e Vinho permitiu a absorção de conhecimentos junto às fontes externas de informação, sejam universidades ou institutos de pesquisas. No entanto, no início da rede, a socialização das informações ainda não se desenvolveu de forma efetiva para todos os atores centrais devido às características organizacionais que os diferenciavam. Principalmente, relacionadas com o modelo de gestão de negócios das vinícolas.

A respeito das considerações gerais sobre o gerenciamento da apropriabilidade da inovação, notou-se a importância da construção de um processo de confiança relacionada com vínculos técnicos e afetivos. Ao mesmo tempo, a existência de procedimentos justos, sem a presença de comportamentos unilaterais, tornou-se uma realidade para a rede a partir da constatação de possibilidades de ganhos diferenciados entre os associados por meio das inovações criadas. Finalmente, no gerenciamento da estrutura da rede, notou-se um relativo consenso no estabelecimento de uma visão de futuro para a rede, associado com relacionamentos que envolveram aspectos técnicos, comerciais, de gestão e amizade.

Esses aspectos demonstram que os mecanismos de coordenação gerenciamento da mobilidade do conhecimento e da estabilidade da rede acabaram ganhando importância decisiva para o sucesso de formação da rede. O gerenciamento efetivo desses mecanismos por meio dos atores centrais analisados, em especial, a Embrapa Uva e Vinho, resultou na principal inovação implementada na rede: a criação da estrutura organizacional denominada Associação dos Produtores de Vinhos Finos do Vale dos Vinhedos. Estrutura essa que foi demandada por meio das exigências do mercado consumidor global por produtos e processos oriundos do setor vitivinícola com alto grau de qualidade e com certificação de procedência. 


\section{Considerações Finais}

Este trabalho teve por objetivo identificar e analisar características estruturais e mecanismos de coordenação envolvidos na formação da rede de cooperação Associação dos Produtores de Vinhos Finos do Vale dos Vinhedos (APROVALE). Assim, com base no conceito de redes visto como estratégia de cooperação, foram analisadas percepções de gestores da rede mencionada e de instituições atuantes no setor vitivinícola brasileiro a respeito do momento de formação da rede APROVALE. Os resultados indicaram, de um modo geral, que a formação da rede como estratégia de cooperação no setor vitivinícola brasileiro possibilitou sucesso no desenvolvimento das organizações associadas, impactando os mercados de inserção regional/nacional, seja por inovações nos produtos, nos processos e na forma de gestão, ou ainda, em mudanças estabelecidas nas relações entre os associados.

Na fase da formação da rede, a influência de forças do ambiente competitivo sobre as organizações do setor vitivinícola, a chegada de multinacionais na Serra Gaúcha, a introdução de castas europeias, a adoção de novas tecnologias de vinificação, constituíram o marco definidor do modelo da estrutura organizacional pretendida. Nessa fase, as redes pessoais e sociais assumiram elevada importância para a formação da rede, bem como para a integração de interesses divergentes em função dos objetivos principais cooperados, sendo construídos relacionamentos técnicos com base em vínculos parentescos.

Por sua vez, os mecanismos de coordenação na fase de formação da rede demonstraram que a diversidade visionária dos atores centrais permitiu a gestão efetiva deles, seja com relação à quantidade de recursos envolvidos, bem como com a sua capacidade de coordenação. Portanto, o gerenciamento de relacionamentos com cunhos técnico e afetivo foi complementar para o sucesso no processo de cooperação neste início.

Os estudos propostos por Ring e Van de Ven (1994) e Dhanaraj e Parkhe (2006) demonstram que o processo de superação de limitações internas e externas vivenciadas pelas redes de cooperação durante sua evolução faz com que organizações associadas modifiquem com sucesso as formas de relacionamentos estabelecidas. Nessa direção, nota-se que na formação da rede APROVALE, a incorporação de novos conhecimentos por meio do compartilhamento e complementariedade de recursos geridos pelos atores centrais tornaram possível a modificação de hábitos de gestão individualizados dos negócios existentes na rede, preservando as diferenças internas das organizações. Isso demonstra a principal contribuição teórica deste estudo ao verificar que estes dois tipos de comportamentos são possíveis, mesmo em uma estrutura de rede de cooperação.

Como estudos futuros são sugeridos a identificação e análise da ação dos mecanismos de coordenação de recursos verificados no processo de formação da rede APROVALE nas demais fases evolutivas dessa rede de cooperação, além da verificação da influência externa que relacionamentos presentes em diferentes contextos sociais e níveis de análise possuem no processo de formação de redes de cooperação em outros setores produtivos.

\section{REFERÊNCIAS}

AHLSTROM-SODERLING, R. SME strategic business networks seen as learning organizations. Journal of Small Business and Enterprise Development, v. 10, n. 4, 2003.

APROVALE. Associação dos Produtores de Vinhos

Finos do Vale dos Vinhedos. [2010]. Disponível em: <www.vv.com.br>. Acesso em: 13 ago. 2010.

ARMSTRONG, J. S. Co-opetition. Journal of

Marketing, v. 61, n. 2, p. 92-99, 1997.

BALESTRIN, A.; VERSCHOORE, J. R.; REYS JR.

E. O campo de estudos sobre redes de cooperação interorganizacional no Brasil. Revista de

Administração Contemporânea, Rio de Janeiro, v. 14, n. 4, p. 458-477, 2010.

BENGTSSON, M.; KOCK, S. Cooperation and competition in relationships between competitors in business networks. The Journal of Business \&

Industrial Marketing, v. 14, n. 3, p. 178-194, 1999. 
BOEHS, C. G. E.; SEGATTO-MENDES, A. P.

Identificação de mecanismos de controle em alianças estratégicas para desenvolvimento tecnológico: um estudo múltiplo de casos no setor metal-mecânico ao longo das fases de relacionamento. Revista de Administração Contemporânea, Rio de Janeiro, v. 11, n. 3, p. 199-221, 2007.

BRASS, D. J. et al. Taking stock of networks and organizations: a multilevel perspective. The Academic Management Journal, v. 47, n. 6, p. 795-817, 2004.

\section{BUTLER, J. E.; PHAN, P.; HANSEN, G. S. Strategic} alliances through Interorganizational networks: a path to entrepreneurial success? Wellesley: College Entrepreneurship Research Conference, 1990.

CHEN, H. H.; LEE, A. H. I.; TONG, Y. New product mix selection for a high technology company in a technology innovation network. Journal of Technology Management in China, v. 1, n. 2, p. 174-189, 2006.

CHUNG, S.; SINGH, H.; LEE, K. Complementarity, status similarity and social capital as drivers of alliance formation. Strategic Management Journal, v. 21, n. 1, 2006.

COLEMAN, J. S. Foundations of social theory. Cambridge: Harvard University Press, 1990.

DHANARAJ, C.; PARKHE, A. Orchestrating innovation networks. Academy of Management Review, v. 31, n. $3,2006$.

EBERS, M.; JARRILLO, C. The construction, forms, and consequences of industry networks. International Studies of Management and Organization, v. 27, p. 3-22, 1997.

\section{EMBRAPA. Empresa Brasileira de Pesquisa}

Agropecuária: dados técnicos. [2010]. Disponível em: <www.embrapa.gov.br>. Acesso em: 10 out. 2010.

FELZENSTEIN, C.; GIMMON, E. Social networks and marketing cooperation in entrepreneurial clusters: an international comparative study. Journal of International Entrepreneurship, v. 7, n. 4, p. 281-291, 2009.
FRANCO, M. J. B. Tipologia de processos de cooperação empresarial: uma investigação empírica sobre o caso português. Revista de Administração Contemporânea Rio de Janeiro, v. 11, n. 3, 2007.

GAMBETTA, D. Can we trust? In: GAMBETTA, D.

(Ed.) Trust: making and breaking cooperative relations. Oxford: Basil Blackwell, 1988.

GERINGER, J. M. Strategic determinants of partner selection criteria in international joint ventures. Journal of International Business Studies, v. 22, n. 1, p. 4161, 1991.

GRANDORI, A. G. An organizational assessment of interfirm coordination modes. Organization Studies, v. 18, n. 6, p. 897-925, 1997.

GULATI, R. Network location an learning: the influence of network resources and firm capabilities on alliance formation. Strategic Management Journal, v. 20, p. 397-425, 1998.

HACKI, R.; LIGHTON, J. The future of the networked company. The McKinsey Quartely, n. 3, p. 26-39, 2001.

HAVNES, P.; SENNESETH, K. A panel study of firm growth among SMEs in networks. Small Business Economics, v. 16, n. 4, p. 293-302, 2001.

HERMOSILLA, A.; SOLÁ, J. Cooperação empresarial. 2. ed. Portugal: IAPMEI, 1991.

HOFFMANN, V. E. et al. Redes de empresas: proposta de uma tipologia para classificação aplicada na indústria de cerâmica de revestimento. Revista de Administração Contemporânea, Rio de Janeiro, v. 11, n. 1, edição especial, p. 103-127, 2007.

HUMAN, S. E.; PROVAN, K. G. An emergent theory of structure and outcomes in small-firm strategic manufacturing network. Academy of Management Journal, v. 40, n. 2, 1997.

IBRAVIN. Instituto Brasileiro do Vinho: dados históricos e recentes do setor vitivinícola brasileiro. [2010]. Disponível em: <http:// www.ibravin.com.br>. Acesso em: 10 maio 2010. 
JARILLO, J. C. On strategic networks. Strategic

Management Journal, v. 9, n. 11, p. 31-41, 1988.

KNOKE, D. Political networks. Cambridge: Cambridge University Press, 1994.

LADO, A. A.; BOYD, N. G.; HANLON, S. C y of Management Review, v. 22, n. 1, p. 110-141, 1997.

LARSON, A. Network dyads in entrepreneurial settings: a study of governance of exchange relationships.

Administrative Science Quartely, v. 37, n. 1, p. 76104, 1992.

LORENZONI, G.; BADEN-FULLER, C. Creating a strategic center to manage a web of partners. California Management Review, v. 37, n. 3, 1995.

MAGALHAES, J. M.; DAUDT, C. G.; PHONLOR, P.

$\mathrm{R}$. Vantagens proporcionadas às pequenas e médias empresas por meio da união em redes de cooperação no contexto do venture capital. Revista de Administração Contemporânea Rio de Janeiro, v. 13, n. 4, p. 583-603, 2009.

OLIVER, A. L. EBERS, M. Networking network studies: an analysis of conceptual configurations in the study of interorganizational relationships. Organization Studies, v. 19, n. 4, p. 549-583, 1998.

RING, P. S.; VAN DE VEN, A. H. Developmental processes of cooperative interorganizational relationships. Academy of Management Journal, v. 19, n. 1, 1994.

PERROW, C. Small-firms networks. In: NOHRIA, N.; ECCLES, R. G. Networks and organizations: structure, form, and action. Cambridge: Harvard University Press, 1992.

PODOLNY, J. M.; STUART, T. E. A role-based ecology of technological change. American Journal of Sociology, Chicago, USA, v. 100, n. 5, p. 1.224-1.260, 1995.

POWELL, W. W. Learning from collaboration: knowledge and networks in the biotechnology and pharmaceutical industries. California Management Review, University of California, Berkeley, v. 40, n. 3, p. 228-240, 1998.

PUTNAM, R. D. Comunidade e democracia e experiência da Itália Moderna. Rio de Janeiro: FGV, 1996.
SCHIBANY, A.; POLT, W. Innovation and networks: an introduction to the theme. In: OCDE (Ed.) Innovative networks: co-operation in national innovation systems. Paris: OCDE, 2001.

SCOTT, J. Social network analysis: a handbook. London: Sage Publications, 2000.

THOMPSON, G.F. Between hierarchies and markets: the logic and limits of network form of organization. New York: Oxford University Press, 2003.

TODEVA, E. Business networks: strategy and structure. New York: Taylor \& Francis, 2006.

VERSCHOORE, J. R.; BALESTRIN, A. Fatores relevantes para o estabelecimento de redes de cooperação entre empresas no Rio Grande do Sul. Revista de

Administração Contemporânea, Rio de Janeiro, v.12, n. 4, p. 1.043-1.069, 2008.

WINKLER, I. Network governance between individual and collective goals. Journal of Leadership and Organizational Studies, v. 12, n. 3, p. 119-134, 2006. 